\title{
Trapping nanoparticles with plasmonic and photonic nanostructures
}

\author{
Kenneth B. Crozier, ${ }^{1,2 *}$ \\ ${ }^{1}$ School of Physics, University of Melbourne, Victoria 3010, Australia \\ 2 Department of Electrical and Electronic Engineering, University of Melbourne, Victoria 3010, Australia \\ Corresponding Author e-mail address: kenneth.crozier@unimelb.edu.au
}

\begin{abstract}
We describe optical trapping with plasmonics and with silicon photonics. We furthermore describe recent work in which fluorescence microscopy is used to track the positions of nanoparticles trapped by structures such as double nanohole apertures.
\end{abstract}

OCIS codes: (140.7010) Laser trapping; (310.6628) Subwavelength structures, nanostructures;

Several compelling advantages exist for the development of integrated approaches for optical trapping, based on photonic or plasmonic structures fabricated on a chip. The first advantage is that chip-based optical traps enable miniaturization of the trapping platform. The second advantage is that the incorporation of sensing modalities is a natural consequence of the chip-based configuration. The third advantage is that strong near-fields can be created by optical nanostructures, boosting the trapping performance.

This presentation describes works by the author and his team in the field of optical trapping with silicon photonics and with plasmonics. The use of silicon microring resonators for trapping (Fig. 1a, [1]) and sensing (Fig. 1b, [2]) particles will be described. We will furthermore describe silicon photonics for sorting particles [3], as well as for sensing proteins [4]. Finally, we will describe experiments in which a silicon photonic crystal cavity trapped a silver nanoparticle on whose surface molecules had been formed. We carried out surface-enhanced Raman spectroscopy (SERS) of these molecules, with the silver nanoparticle held in position via the photonic crystal cavity (Fig. 1c, [5]).

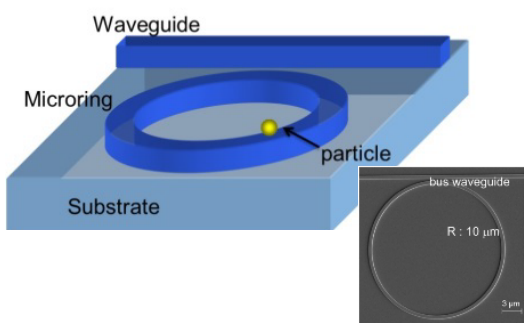

a).

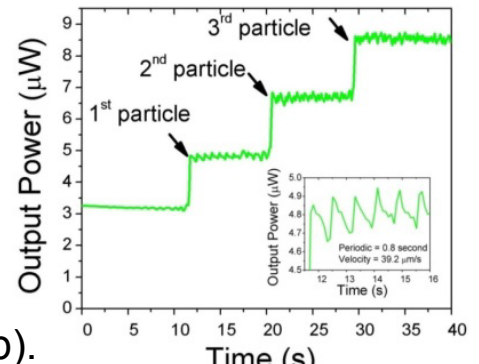

b). Time (s) c).

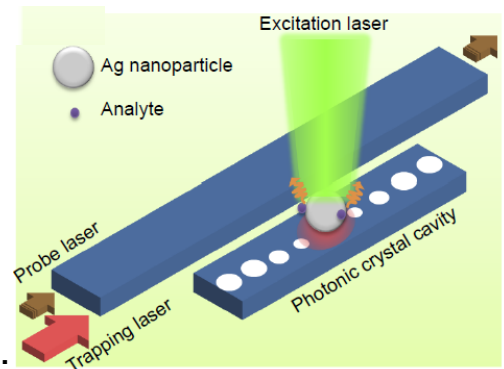

Fig.1. (a) Top: schematic illustration of optical trapping of microparticle with Si microring resonator. Bottom: scanning electron microscope image of fabricated Si microring and bus waveguide. From [1]. b). Output power in waveguide coupled to Si microring resonator. No particles are trapped initially (time $=0 \mathrm{~s}$ ). Output power increases in step-like fashion with the trapping of each additional particle. From [2]. c). Schematic illustration of trapping of $\mathrm{Ag}$ nanoparticle with Si photonic crystal resonator. Pump and probe lasers (wavelengths are near $1550 \mathrm{~nm}$ ) provide light for trapping nanoparticles and monitoring cavity resonance wavelength, respectively. SERS signals from molecules on Ag nanoparticle surface are excited with green excitation laser, and collected with an optical microscope into a spectrometer (not shown). From [5]

Plasmonic nanostructures are compelling for optical trapping due to the large gradient forces they can generate, a consequence of their ability to generate highly confined optical fields. Yet deleterious thermal effects can also occur. We describe the use of a plasmonic nanotweezer with an integrated heat-sink (Fig. 2, [6]). If time permits, we will also describe recent work in which fluorescence microscopy was used to track the position of a nanoparticle trapped by a double nanohole aperture [7]. 

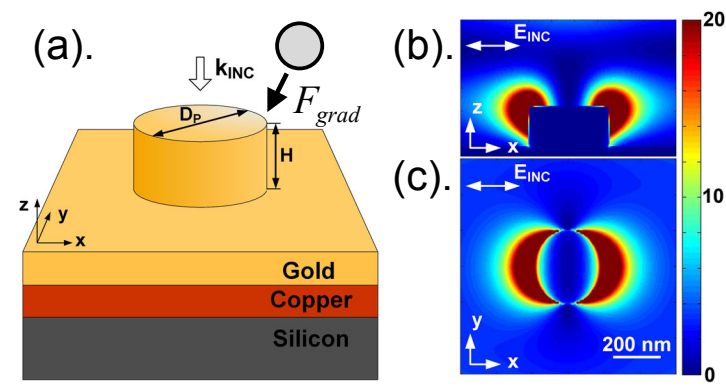

(d).

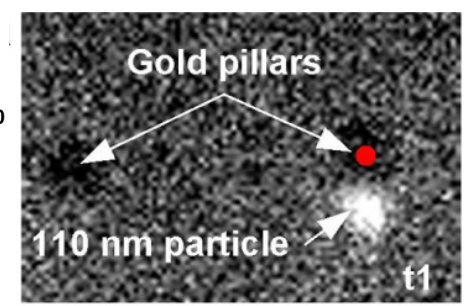

(e).

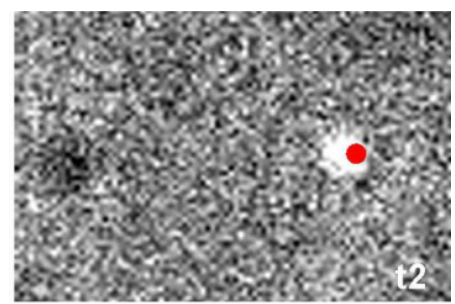

Fig.2. a). Plasmonic nanotweezer. $\boldsymbol{D}_{\boldsymbol{P}}: 280 \mathrm{~nm} . \boldsymbol{H}: 130 \mathrm{~nm}$ [6]. Simulation of electric field intensity $\left(\mathrm{E}^{2}\right)$ on b). cross-section of nanopillar, c). topview of nanopillar. CCD camera images of particle d). before and e). after trapping. Nanopillar position indicated by red dot.

\section{References}

[1] S. Lin, E. Schonbrun, and K. Crozier, "Optical Manipulation with Planar Silicon Microring Resonators," Nano Letters 10, 2408 (2010)

[2] S. Lin and K.B. Crozier, "Planar silicon microrings as wavelength-multiplexed optical traps for storing and sensing particles," Lab on a Chip 11, 4047-4051 (2011);

[3] S. Lin and K.B. Crozier, "An integrated microparticle sorting system based on near-field optical forces and a structural perturbation," Optics Express 20, 3367-3374 (2012);

[4] S. Lin and K.B. Crozier, "Trapping-Assisted Sensing of Particles and Proteins using On-Chip Optical Microcavities," ACS Nano 7, 1725 (2013)

[5] S. Lin, W. Zhu, Y. Jin and K.B. Crozier, "Surface Enhanced Raman Scattering with Ag Nanoparticles Optically Trapped by a Photonic Crystal Cavity," Nano Letters 13, 559 (2013)

[6] K. Wang, E. Schonbrun, P. Steinvurzel and K.B. Crozier, "Trapping and rotating nanoparticles using a plasmonic nano-tweezer with an integrated heat sink," Nature Communications vol. 2, 469 (2011)

[7] Z. Xu, W. Song and K.B. Crozier, "Optical tracking of a nanoparticle trapped by a double nanohole aperture", in Conference on Lasers and Electro-Optics, OSA Technical Digest (online) (Optical Society of America, 2016), paper FM2B.6. 